\title{
Isovector channel of quark-meson-coupling model and its effect on symmetry energy
}

\author{
X.B. Wang ${ }^{\mathrm{a}}$, C. Qi ${ }^{\mathrm{b}}$, F.R. Xu $\mathrm{u}^{\mathrm{a}, \mathrm{c}, *}$ \\ ${ }^{a}$ School of Physics, and State Key Laboratory of Nuclear Physics and Technology, Peking \\ University, Beijing 100871, China \\ ${ }^{b}$ KTH (Royal Institute of Technology), Alba Nova University Center, SE-10691 Stockholm, \\ Sweden \\ ${ }^{c}$ Center for Theoretical Nuclear Physics, National Laboratory for Heavy Ion Physics, \\ Lanzhou 730000, China
}

\begin{abstract}
The non-relativistic approximation of the quark-meson-coupling model has been discussed and compared with the Skyrme-Hartree-Fock model which includes spin exchanges. Calculations show that the spin-exchange interaction has important effect on the descriptions of finite nuclei and nuclear matter through the Fock exchange. Also in the quark-meson-coupling model, it is the Fock exchange that leads to a nonlinear density-dependent isovector channel and changes the density-dependent behavior of the symmetry energy.
\end{abstract}

Keywords:

quark-meson-coupling model, Skyrme force, Energy density functional, Nuclear matter, Finite nuclei, Symmetry energy

\section{Introduction}

Both relativistic and non-relativistic energy density functional models have been developed to describe the many-body system of atomic nucleus [1]. According to the Kohn-Sham theorem [2], models leading to the same energy functional are equivalent as far as physical results are concerned [3]. Indeed, systematical calculations have shown that both relativistic and non-relativistic models give quite similar results for the ground-state properties and low-energy dynamics of stable nuclei [4]. However, significant differences are found when calculations go to the extreme cases of high isospin and high density [1, 5]. The origin of these differences is still not clear.

In the non-relativistic approach, the Skyrme-Hartree-Fock (SHF) model has been extensively employed in the investigation of nuclear structure [1, 4] since the first work of Vautherin and Brink [6]. The Skyrme potential contains ten

\footnotetext{
${ }^{*}$ Corresponding author

Email address: frxu@pku.edu.cn (F.R. Xu)
}

Preprint submitted to Elsevier

March 1, 2022 
parameters, but only six of the parameters have been well determined [7]. This leads to many sets of parameters with different emphases on the descriptions of nuclear properties [8, 9]. It was tested by Stone et al. [8] that about one third of existing Skyrme parameter sets give the monotonic increase of the symmetry energy with density while other two third give the decrease of the symmetry energy after certain supra-saturation density. It should be mentioned that predictions for the density dependence of the symmetry energy depend strongly on models and parameters employed [5, 10, 11]. As examples, the VariationalMany-Body [12 14] and Dirac-Brueckner-Hartree-Fock [15] theories predict that the symmetry energy turns to decrease after certain supra-saturation density, while RMF [16, 17] and Brueckner-Hartree-Fock [18] models present that the symmetry energy increases monotonically with increasing density. Therefore, it is encouraging to investigate possible physics origins for the different behaviors of the symmetry energy at high densities [5, 19 21] which may shed light on our understanding of nuclear force.

Based on the mean-field approximation but including the response of the quark structure of the nucleon in the nuclear environment, the relativistic quarkmeson-coupling model (QMC) was proposed by Guichon et al. [22, 23]. In Ref. [24] an interesting similarity was found by comparing the energy density functionals of the SHF model and the non-relativistic approximation of QMC models, providing an understanding of the physical origin of the Skyrme force within the relativistic approach. With the restriction of $N=Z$, the authors [24] obtained a set of parameters of the Skyrme force that gives reasonable descriptions of nuclear matter and finite nuclei. The work suggests that investigations regarding the physics of effective nucleon-nucleon interactions remain interesting. In Ref. [24], the Skyrme spin-exchange terms that involve the $x_{1}, x_{2}$, and $x_{3}$ parameters are neglected. The present discussion will include the spin-exchange terms. It will be shown that these terms have important effects on nuclear properties at high isospin and high density, particularly on the density dependence of the symmetry energy.

\section{Energy density functionals of QMC and Skyrme Hartree-Fock mod- els}

The QMC model gives a relativistic quark-level description of nuclear matter [22, 23]. In the model, a baryon in nuclear medium is assumed to be a non-overlapping static spherical bag in which quarks are coupled to meson fields in the mean-field approximation [22 25]. For modeling, one can choose an isoscalar-scalar field $(\sigma)$ for the medium-range attraction, an isoscalar-vector field $(\omega)$ for the short-range repulsion, and an isovector-vetor field $(\rho)$ for isospin channel [24]. Detailed discussions on the Hartree-Fock (HF) approximation of the QMC model can be found in Refs. [24, 26 28].

As has been given in Ref. 24], for finite nuclei, the non-relativistic HF approximation of the QMC density functional can be written as follows

$$
\mathcal{E}^{\mathrm{QMC}}=\rho M+\frac{\tau}{2 M}+\mathcal{H}_{0}+\mathcal{H}_{3}+\mathcal{H}_{\mathrm{eff}}+\mathcal{H}_{\mathrm{fin}}+\mathcal{H}_{\mathrm{so}}
$$


with

$$
\begin{aligned}
\mathcal{H}_{0}+\mathcal{H}_{3}= & \rho^{2}\left[\frac{-3 G_{\rho}}{32}+\frac{G_{\sigma}}{8\left(1+d \rho G_{\sigma}\right)^{3}}-\frac{G_{\sigma}}{2\left(1+d \rho G_{\sigma}\right)}+\frac{3 G_{\omega}}{8}\right] \\
& +\left(\rho_{n}-\rho_{p}\right)^{2}\left[\frac{5 G_{\rho}}{32}+\frac{G_{\sigma}}{8\left(1+d \rho G_{\sigma}\right)^{3}}-\frac{G_{\omega}}{8}\right], \\
\mathcal{H}_{\mathrm{eff}}= & \left(\frac{G_{\rho}}{4 m_{\rho}^{2}}+\frac{G_{\sigma}}{2 M^{2}}\right) \rho \tau \\
& +\left(-\frac{G_{\rho}}{8 m_{\rho}^{2}}-\frac{G_{\sigma}}{2 m_{\sigma}^{2}}+\frac{G_{\omega}}{2 m_{\omega}^{2}}-\frac{G_{\sigma}}{4 M^{2}}\right)\left(\rho_{n} \tau_{n}+\rho_{p} \tau_{p}\right), \\
\mathcal{H}_{\mathrm{fin}}= & \left(\frac{-3 G_{\rho}}{16 m_{\rho}^{2}}-\frac{G_{\sigma}}{2 m_{\sigma}^{2}}+\frac{G_{\omega}}{2 m_{\omega}^{2}}-\frac{G_{\sigma}}{4 M^{2}}\right) \rho \nabla^{2} \rho \\
+ & \left(\frac{9 G_{\rho}}{32 m_{\rho}^{2}}+\frac{G_{\sigma}}{8 m_{\sigma}^{2}}-\frac{G_{\omega}}{8 m_{\omega}^{2}}+\frac{G_{\sigma}}{8 M^{2}}\right)\left(\rho_{n} \nabla^{2} \rho_{n}+\rho_{p} \nabla^{2} \rho_{p}\right),
\end{aligned}
$$

and

$$
\begin{aligned}
\mathcal{H}_{\text {so }}= & \left(\frac{-G_{\sigma}}{4 M^{2}}+\frac{G_{\omega}\left(1-2 \mu_{s}\right)}{4 M^{2}}\right) \rho \nabla \cdot J \\
& +\left(\frac{-G_{\sigma}}{8 M^{2}}+\frac{G_{\omega}\left(1-4 \mu_{s}\right)}{8 M^{2}}-\frac{3 G_{\rho}\left(-1+2 \mu_{v}\right)}{32 M^{2}}\right) \\
& \times\left(\rho_{n} \nabla \cdot J_{n}+\rho_{p} \nabla \cdot J_{p}\right),
\end{aligned}
$$

where $M$ is for the mass of the nucleon, $\tau=\tau_{n}+\tau_{p}$ for kinetic energy density, and $\rho=\rho_{n}+\rho_{p}$ for density with $n$ and $p$ denoting the neutron and proton, respectively. $G_{\sigma}=g_{\sigma}^{2} / m_{\sigma}^{2}, G_{\omega}=g_{\omega}^{2} / m_{\omega}^{2}$ and $G_{\rho}=g_{\rho}^{2} / m_{\rho}^{2}$ are defined with $g_{\sigma}$, $g_{\omega}, g_{\rho}$ for coupling constants and $m_{\sigma}, m_{\omega}, m_{\rho}$ for the masses of the mesons. The parameter $d$ is the scalar polarizability of the nucleon 24]. In the numerical calculations of the present work, we take $d=0.150 \mathrm{fm}$ from Ref. [24]. $\mu_{s}$ and $\mu_{v}$ are isoscalar and isovector magnetic moments of the nucleon, respectively. $\mu_{s}=\mu_{p}+\mu_{n}$ and $\mu_{v}=\mu_{p}-\mu_{n}\left(\mu_{p}=2.79 \mu_{\mathrm{N}}, \mu_{n}=-1.91 \mu_{\mathrm{N}}\right) . J_{n}$ and $J_{p}$ are the spin density of the neutron and proton, respectively, with the total spin density of $J=J_{n}+J_{p}$.

In Refs. 24, 29] it was shown that the six elementary parameters $\left(t_{0}, t_{1}, t_{2}\right.$, $t_{3}, x_{0}$ and $W_{0}$ ) of the Skyrme force can be understood in the framework of the QMC model. In the present work, we study in a more detailed energy density functional of the SHF model with spin-exchange parameters $\left(x_{1}, x_{2}, x_{3}\right)$ which can produce effects through the Fock exchange. We can have a general form of the Skyrme energy density functional as [30],

$$
\begin{aligned}
\mathcal{H}_{0}+\mathcal{H}_{3}= & \rho^{2}\left[\frac{t_{3} \rho^{\alpha}}{16}+\frac{3 t_{0}}{8}\right] \\
& +\left(\rho_{n}-\rho_{p}\right)^{2}\left[-\frac{t_{0}\left(2 x_{0}+1\right)}{8}-\frac{\left(2 x_{3}+1\right) t_{3} \rho^{\alpha}}{48}\right],
\end{aligned}
$$




$$
\begin{aligned}
\mathcal{H}_{\mathrm{eff}}= & \frac{1}{8}\left[\left(2+x_{1}\right) t_{1}+\left(2+x_{2}\right) t_{2}\right] \rho \tau \\
& +\frac{1}{8}\left[t_{2}\left(2 x_{2}+1\right)-t_{1}\left(2 x_{1}+1\right)\right]\left(\rho_{n} \tau_{n}+\rho_{p} \tau_{p}\right), \\
\mathcal{H}_{\text {fin }}=\quad- & \frac{1}{32}\left[3 t_{1}\left(2+x_{1}\right)-t_{2}\left(2+x_{2}\right)\right] \rho \nabla^{2} \rho \\
& +\frac{1}{32}\left[3 t_{1}\left(2 x_{1}+1\right)+t_{2}\left(2 x_{2}+1\right)\right]\left[\rho_{n} \nabla^{2} \rho_{n}+\rho_{p} \nabla^{2} \rho_{p}\right],
\end{aligned}
$$

and

$$
\mathcal{H}_{\text {so }}=-\frac{1}{2} W_{0}\left(\rho \nabla \cdot J+\rho_{n} \nabla \cdot J_{n}+\rho_{p} \nabla \cdot J_{p}\right) .
$$

The QMC and SHF models have the same density-dependent forms in the effective-mass term $\mathcal{H}_{\text {eff }}$ and the finite-range term $\mathcal{H}_{\text {fin }}$. By comparing $\mathcal{H}_{\text {eff }}$ and $\mathcal{H}_{\text {fin }}$, we obtain the following relations,

$$
\begin{gathered}
t_{1}=\frac{G_{\rho}}{2 m_{\rho}^{2}}+\frac{3 G_{\sigma}}{2 M^{2}}-\frac{2 G_{\omega}}{m_{\omega}^{2}}+\frac{2 G_{\sigma}}{m_{\sigma}^{2}}, \\
x_{1}=\frac{2 \frac{G_{\rho}}{m_{\rho}^{2}}}{\frac{G_{\rho}}{m_{\rho}^{2}}+\frac{3 G_{\sigma}}{M^{2}}-\frac{4 G_{\omega}}{m_{\omega}^{2}}+\frac{4 G_{\sigma}}{m_{\sigma}^{2}}}, \\
t_{2}=-\frac{G_{\rho}}{2 m_{\rho}^{2}}+\frac{5 G_{\sigma}}{6 M^{2}}+\frac{2 G_{\omega}}{m_{\omega}^{2}}-\frac{2 G_{\sigma}}{m_{\sigma}^{2}},
\end{gathered}
$$

and

$$
x_{2}=\frac{\frac{G_{\rho}}{m_{\rho}^{2}}-\frac{2 G_{\sigma}}{3 M^{2}}}{-\frac{G_{\rho}}{2 m_{\rho}^{2}}+\frac{5 G_{\sigma}}{6 M^{2}}+\frac{2 G_{\omega}}{m_{\omega}^{2}}-\frac{2 G_{\sigma}}{m_{\sigma}^{2}}} .
$$

Comparing the energy density functionals of the QMC and SHF models, we find that main differences in density dependence appear in the terms which involve the parameters $t_{3}$ and $x_{3}$ in the zero-range term $\mathcal{H}_{0}$ and the densitydependent term $\mathcal{H}_{3}$. For a qualitative study, we expand the $\mathcal{H}_{0}+\mathcal{H}_{3}$ term around the saturation density in respect that the density range of finite nuclei is around the saturation density. For the coefficient of the $\rho^{2}$ term in the corresponding $\mathcal{H}_{0}+\mathcal{H}_{3}$ term, the first and second orders of the expansions give

$$
\frac{-3 G_{\rho}}{32}+\frac{G_{\sigma}}{8\left(1+d \rho_{0} G_{\sigma}\right)^{3}}-\frac{G_{\sigma}}{2\left(1+d \rho_{0} G_{\sigma}\right)}+\frac{3 G_{\omega}}{8}=\frac{3 t_{0}}{8}+\frac{t_{3} \rho_{0}^{\alpha}}{16},
$$

and

$$
-\frac{G_{\sigma}}{8} \frac{3 d \rho_{0} G_{\sigma}}{\left(1+d \rho_{0} G_{\sigma}\right)^{4}}+\frac{G_{\sigma}}{2} \frac{d \rho_{0} G_{\sigma}}{\left(1+d \rho_{0} G_{\sigma}\right)^{2}}=\frac{t_{3}}{16} \alpha \rho_{0}{ }^{\alpha}
$$


where $\rho_{0}$ is the saturation density. Similarly, the expansions of the coefficients of the $\left(\rho_{n}-\rho_{p}\right)^{2}$ term give the following relations,

$$
\frac{5 G_{\rho}}{32}+\frac{G_{\sigma}}{8\left(1+d \rho_{0} G_{\sigma}\right)^{3}}-\frac{G_{\omega}}{8}=-\frac{\left(2 x_{3}+1\right) t_{3} \rho_{0}^{\alpha}}{48}-\frac{t_{0}\left(2 x_{0}+1\right)}{8},
$$

and

$$
\frac{G_{\sigma}}{8} \frac{3 d \rho_{0} G_{\sigma}}{\left(1+d \rho_{0} G_{\sigma}\right)^{4}}=\frac{\left(2 x_{3}+1\right)}{48} t_{3} \alpha \rho_{0}{ }^{\alpha} .
$$

From Eqs. (15) and (17), the parameter $x_{3}$ can be obtained immediately. The parameters $x_{0}, t_{0}$ and $t_{3}$ are related to the parameter $\alpha$. We can use the symmetry energy of the nuclear matter at the saturation density to determine the parameter $\alpha$. Fig. 11 plots the variation of the symmetry energy with changing the parameter $\alpha$. At each given $\alpha$ value, the parameters $x_{0}, t_{0}$ and $t_{3}$ are obtained using Eqs. (14)-(16), and the SHF symmetry energy [30] is calculated with these parameters including those given by Eqs. (10)-(13). The QMC parameters are taken from Ref. 24]. It is well accepted that the symmetry energy of the nuclear matter at the saturation density is about $30 \mathrm{MeV}$, which leads to an $\alpha$ value of about $1 / 6$, that is consistent with the usual value in the SHF model, see Fig. 1. With the $\alpha$ value and the QMC parameters we can determine the $x_{0}, t_{0}$ and $t_{3}$ using Eqs. (14)-(16).

For completeness, we take the $W_{0}$ parameter corresponding to the spin-orbit term $\mathcal{H}_{\text {so }}$ as Ref. 24]

$$
W_{0}=\frac{1}{12 M^{2}}\left[5 G_{\sigma}-5 G_{\omega}\left(1-2 \mu_{s}\right)-\frac{3}{4} G_{\rho}\left(1-2 \mu_{v}\right)\right] .
$$

With the QMC parameters at $m_{\sigma}=700 \mathrm{MeV}$ [24], all the Skyrme parameters including $x_{1}, x_{2}$ and $x_{3}$ have been derived, listed in Table 1 Similar to the result given in Ref. 24], the obtained values of the $x_{0}, t_{0}, t_{1}, t_{2}, t_{3}$ and $W_{0}$ parameters are close to the $\mathrm{SkM}^{*}$ force. In Ref. [24], the parameters $x_{1}, x_{2}$ and $x_{3}$ are set to be zero. However, it was pointed out that the parameter $x_{3}$ has significant effect on the symmetry energy [31]. There already exist many sets of Skyrme parameters. It is not our aim to derive a new set of parameters. In the present work, we extend the understanding of the Skyrme force in the framework of the QMC model, which has been done primarily in Ref. [24].

\section{Calculations and discussions}

As the first step, we compare the Hartree-Fock calculations of the QMC and Skyrme models with different parameters for the nuclear matter, shown in fig. 2. It is found that the calculations give similar properties of the nuclear matter around the saturation density. However, different behaviors are seen in densities away from the saturation. The Sqmc parameters which contain the spin-exchange parameters $x_{1}, x_{2}$ and $x_{3}$ give a similar behavior of the symmetry 
Table 1. The Skyrme parameters obtained in the present work (labeled by Sqmc) from the QMC model [24].

\begin{tabular}{lr}
\hline Skyrme Parameters & \\
\hline$t_{0}\left(\mathrm{MeV} \mathrm{fm}^{3}\right)$ & -2648.19 \\
$t_{1}\left(\mathrm{MeV} \mathrm{fm}^{5}\right)$ & 371.07 \\
$t_{2}\left(\mathrm{MeV} \mathrm{fm}^{5}\right)$ & -121.644 \\
$t_{3}\left(\mathrm{MeV} \mathrm{fm}^{4}\right)$ & 15553.495 \\
$x_{0}$ & 0.60146 \\
$x_{1}$ & 0.2697 \\
$x_{2}$ & -0.23701 \\
$x_{3}\left(\mathrm{MeV} \mathrm{fm}^{5}\right)$ & 0.6968 \\
$W_{0}$ & 104.498 \\
$\alpha$ & $1 / 6$ \\
\hline
\end{tabular}

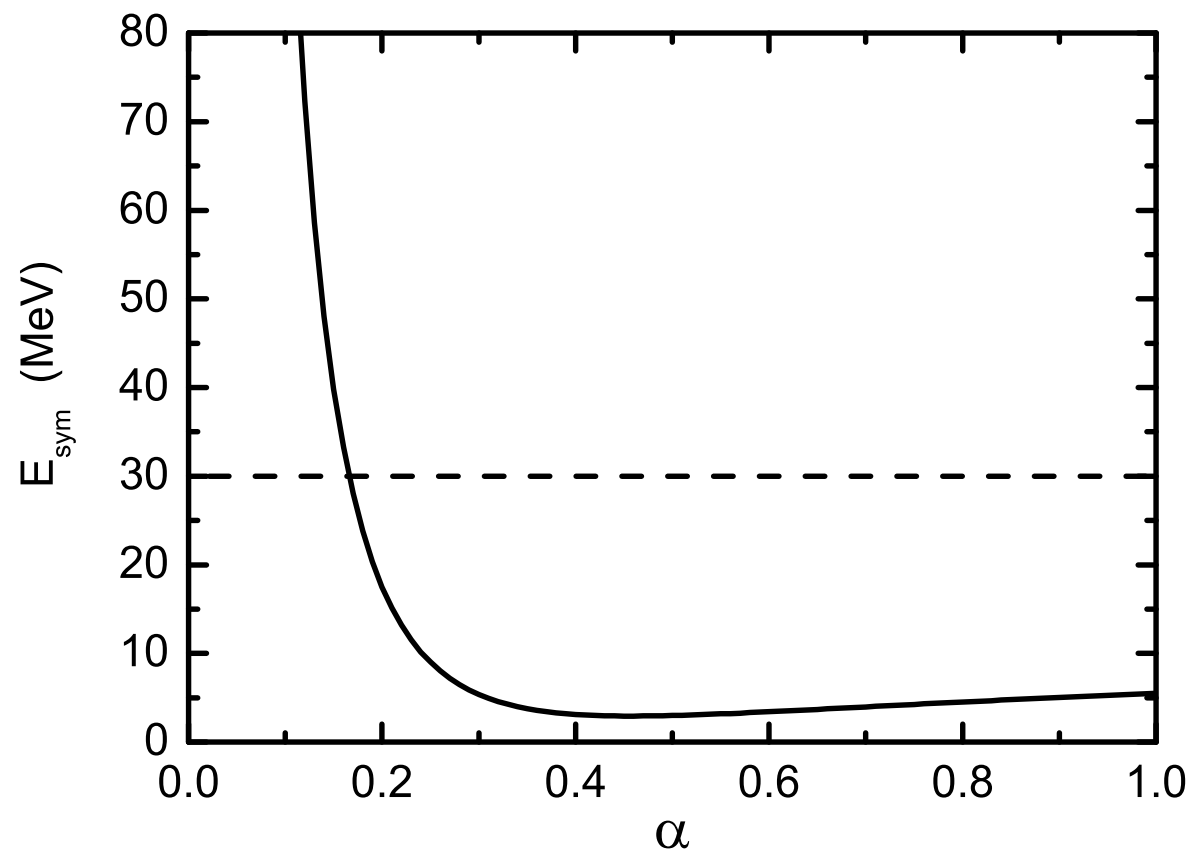

Figure 1. The symmetry energy for nuclear matter at the saturation density as a function of the $\alpha$ parameter, calculated using the SHF model with the parameters obtained through the QMC model. The dashed line indicates the commonly-adopted symmetry energy of $a_{\mathrm{s}}=30$ $\mathrm{MeV}$. 

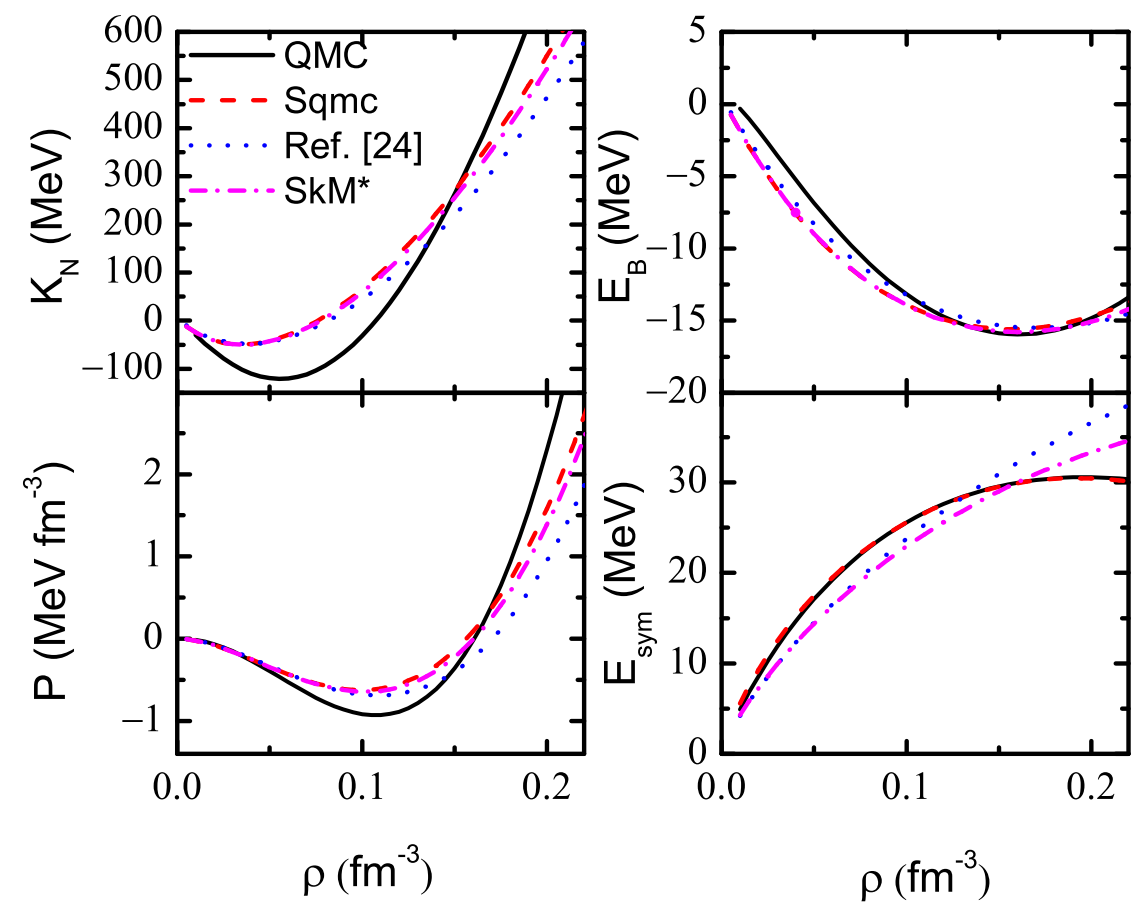

Figure 2. (Color online) Comparisons between the QMC and Skyrme calculations of the properties of the nuclear matter, i.e., incompressibility $K_{\mathrm{N}}$, pressure $P$, binding energy $E_{\mathrm{B}}$ and symmetry energy $E_{\text {sym }}$ within the Hartree-Fock approximation. For the SHF calculations, $\mathrm{SkM}^{*}$, Sqmc and the Skyrme parameters given in Ref. 24] have been used.

energy against density as the QMC model. The symmetry energy curve calculated by the Skyrme force neglecting the $x_{1}, x_{2}$ and $x_{3}$ parameters [24] is much more stiff than that obtained by the QMC model. A soft symmetry energy against density seems more acceptable from the recent analysis of experimental data [11].

As a further test, we calculate the properties of finite nuclei by the Sqmc parameters. We use the SHF-BCS code of ev8 [32] with a self-consistent two-body center-of-mass correction. Calculations for the double magic nuclei have shown a significant improvement, compared with those in Ref. 24] where the parameters $x_{1}, x_{2}$ and $x_{3}$ were set zero. We have also calculated binding energies for the long chain of $\mathrm{Sn}$ isotopes from ${ }^{100} \mathrm{Sn}$ to ${ }^{132} \mathrm{Sn}$, for which the paring strength is fitted by reproducing the neutron separation energies of Sn isotopes, shown in Fig. 3. We see that reasonable agreements between data and calculations are obtained with the Sqmc parameters. Here we should remind that the Sqmc parameters are obtained by comparing the energy density functionals of the QMC and SHF models for the nuclear matter without fitting the properties of 


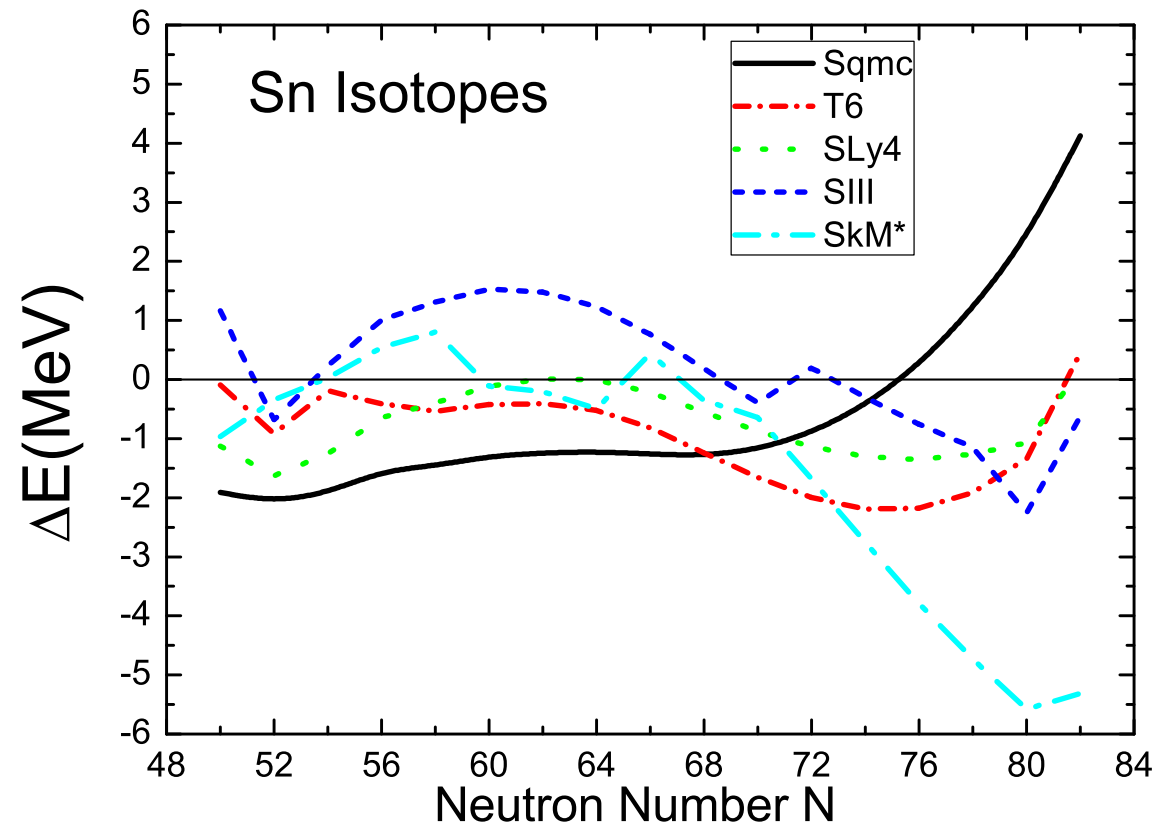

Figure 3. (Color online) Difference between experimental and calculated binding energies for even-even Sn isotopes. The calculations are done using SHF+BCS with different sets of Skyrme parameters. 


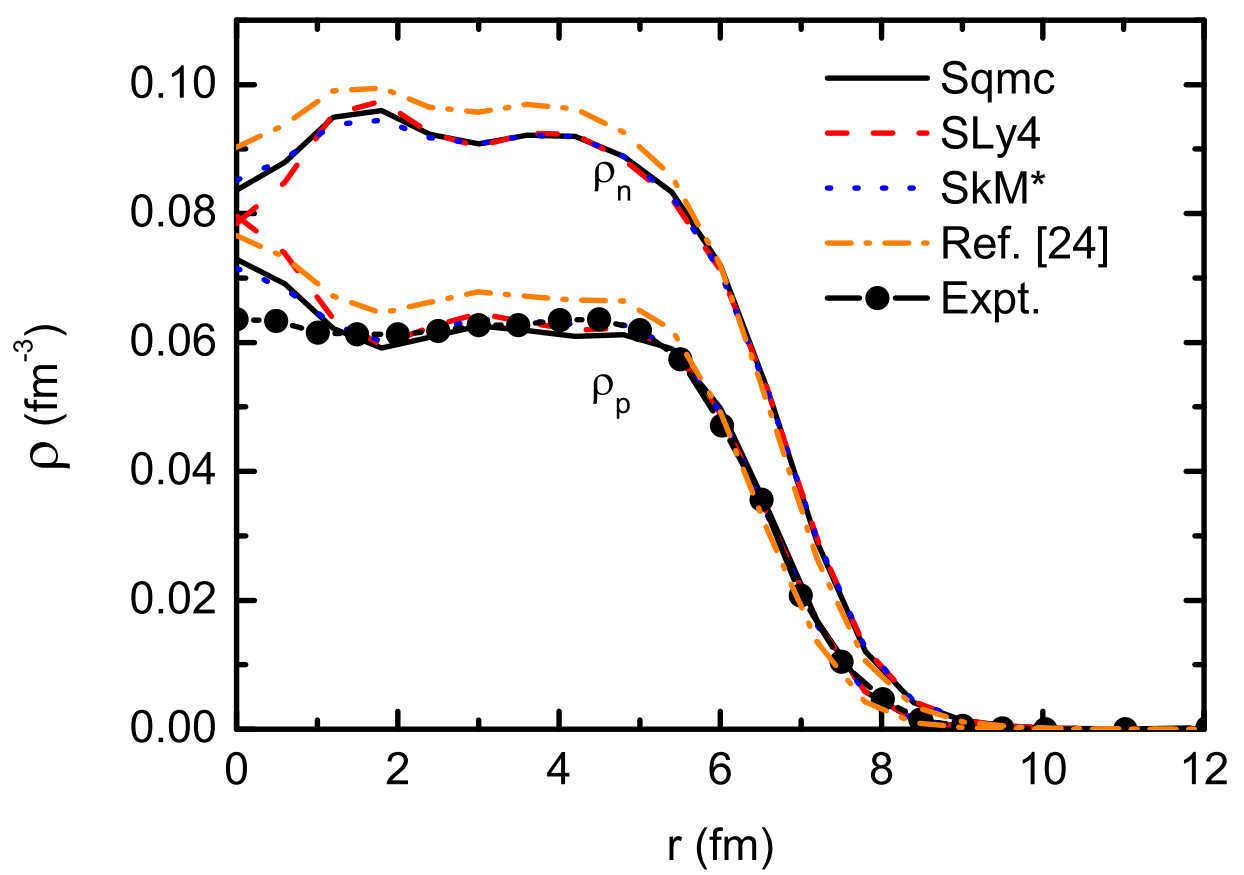

Figure 4. (Color online) Neutron and proton density distributions for ${ }^{208} \mathrm{~Pb}$ (indicated by $\rho_{n}$ and $\rho_{p}$, respectively) calculated with Sqme, SLy4, SkM* and the Skyrme parameters given in Ref. [24], compared with the experimental charge density [33]. 
finite nuclei, while other sets of the Skyrme parameters were determined by the fitting procedure to the experimental data of finite nuclei (including the data of binding energies).

Fig. 4 shows the calculated neutron and proton density distributions for ${ }^{208} \mathrm{~Pb}$, compared with the experimental charge density [33]. The Sqme force gives a similar density distribution to those obtained by the SLy4 and SkM* forces, agreeing well with the data. We see that the calculations with the inclusion of the spin-exchange terms are improved noticeably.

In mean-field models, the concept of the effective mass of the nucleon mainly describes effects related to the nonlocality of the underlying nuclear interactions and the Pauli exchange effects in nuclear system [34]. The effective mass for the nuclear matter in the SHF model can be written as [30]

$$
\begin{aligned}
\frac{\hbar^{2}}{2 M_{f}^{*}} \tau_{f}=\quad & \tau_{f}\left\{\frac{\hbar^{2}}{2 M}+\frac{1}{8} \rho\left[t_{1}\left(2+x_{1}\right)+t_{2}\left(2+x_{2}\right)\right]\right. \\
& \left.+\frac{1}{8} \rho_{f}\left[t_{2}\left(1+2 x_{2}\right)-t_{1}\left(1+2 x_{1}\right)\right]\right\},
\end{aligned}
$$

where the index " $f$ " indicates the proton or neutron. In the QMC model, the non-relativistic effective mass can be extracted when one collects the $\rho \tau$ terms,

$$
\begin{aligned}
\frac{\hbar^{2}}{2 M_{f}^{*}} \tau_{f}=\quad & \tau_{f}\left\{\frac{\hbar^{2}}{2 M}+\frac{1}{2} \frac{G_{\sigma}}{M^{2}} \frac{\rho}{1+d G_{\sigma} \rho}+\frac{1}{4} \frac{G_{\rho}}{m_{\rho}^{2}} \rho\right. \\
& \left.+\rho_{f}\left(-\frac{G_{\rho}}{8 m_{\rho}^{2}}-\frac{G_{\sigma}}{2 m_{\sigma}^{2}}+\frac{G_{\omega}}{2 m_{\omega}^{2}}-\frac{G_{\sigma}}{4 M^{2}}\right)\right\} .
\end{aligned}
$$

The first two terms are the Hartree contribution while the remaining terms come from the Fock exchange.

In the SHF model, the effect from spin-exchange terms with the parameters $x_{1}, x_{2}$ and $x_{3}$ appear explicitly in the effective mass of Eq. (19). For the QMC model, if the effect from the Fock exchange is neglected, the non-relativistic effective mass contains only the isovector term. (The isovector effective mass can be obtained by setting $\rho_{f}=0$ in Eq. (20).) If the Fock term is taken into account, the $\rho$ meson has contribution to both the isovector and isoscalar effective mass. The non-relativistic effective mass calculated by the QMC model for the symmetric nuclear matter is shown in Fig. 5.

The longstanding problem remains about the neutron-proton effective mass splitting, which is crucial for extracting the density dependence of symmetry energy from nuclear reaction using transport models [5, 35]. The dominant contribution to the neutron-proton effective mass splitting is from the isovector channel of the energy density functional. Fig. 6 gives the changes of the effective masses with the isospin, compared with SHF calculations. The behavior of effective masses in the QMC calculations is similar to that of the SkM* calculations. It seems more acceptable recently that the effective mass of neutron is bigger than that of proton [36, 37]. 


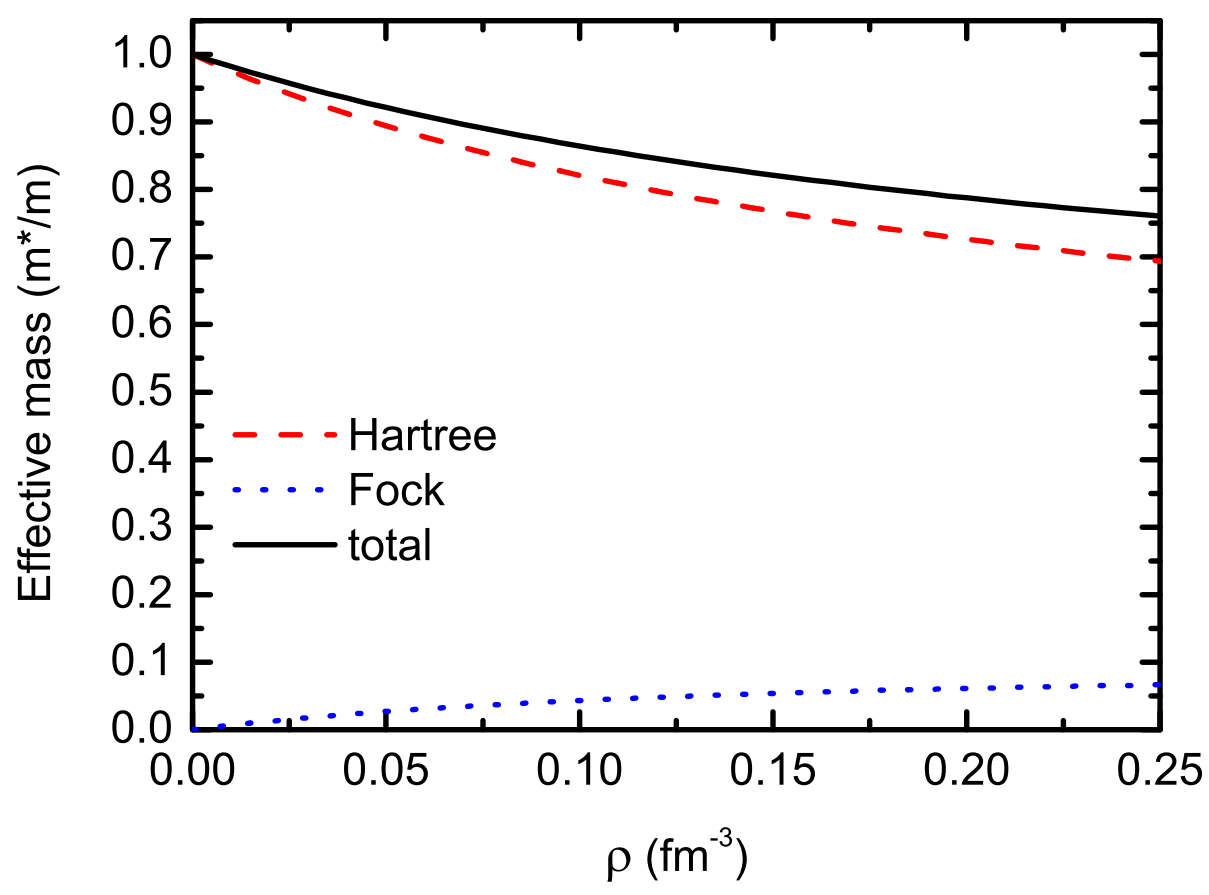

Figure 5. (Color online) The density dependence of the non-relativistic effective mass, $m^{*} / m$, calculated by the QMC model for the symmetric nuclear matter. 


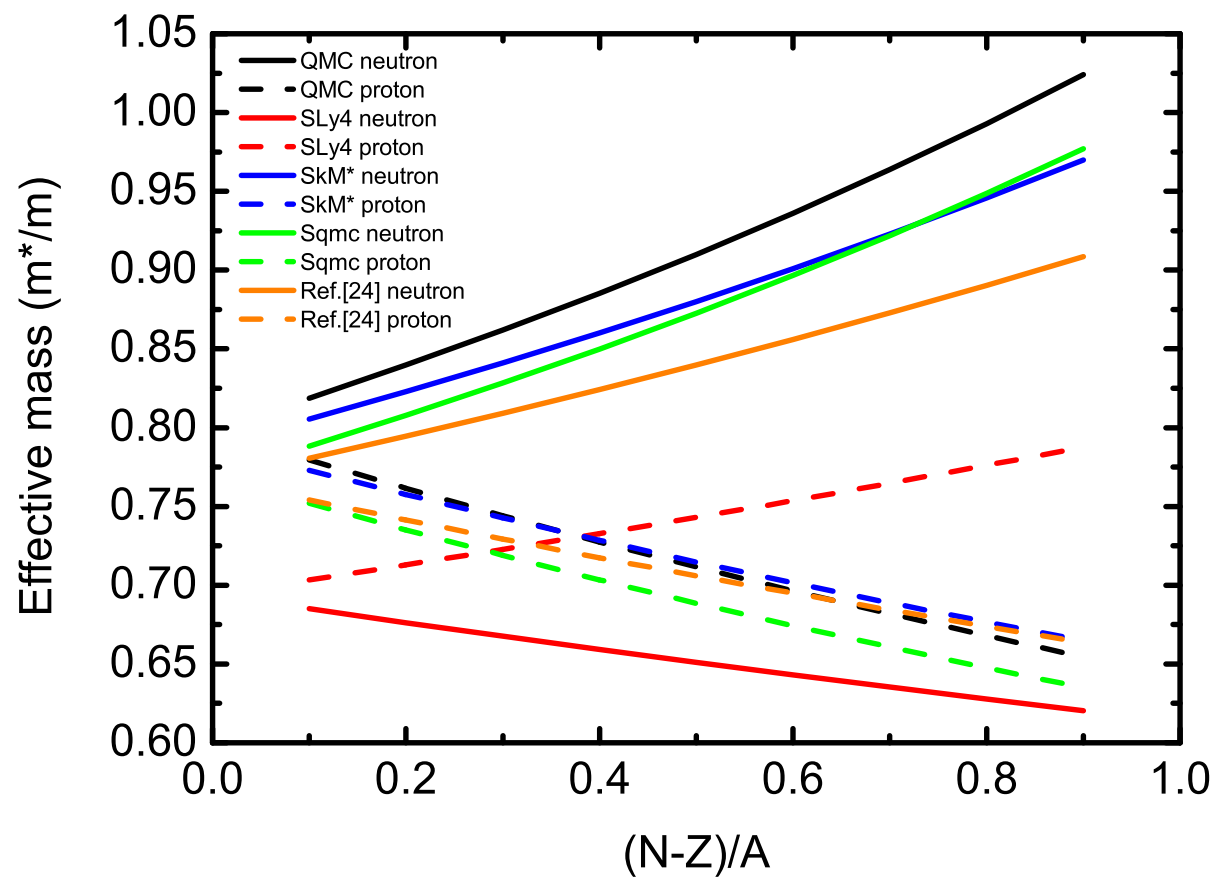

Figure 6. (Color online) Calculated non-relativistic effective masses $\left(\mathrm{m}^{*} / \mathrm{m}\right)$ of the proton and neutron against the isospin in the QMC calculations, compared with the SHF calculations with Sqmc, SLy4, SkM* and the Skyrme parameters given in Ref. 24]. The calculations are done at the saturation density of $\rho=0.16 \mathrm{fm}^{-3}$. 
In Ref. [38] it was shown that the three-body term has an important influence on the symmetry energy curves. In Fig. 2, we have already given the symmetry energy for the nuclear matter, calculated by the QMC and SHF models within the Hartree-Fock approximation. In order to gain a more detailed insight into the nature of the symmetry energy and the effect from the Fock exchange, we make further analysis. The QMC symmetry energy for the nuclear matter within the Hartree approximation is written as,

$$
a_{\text {sym }}^{\mathrm{H}}=\frac{\hbar^{2}}{6 M}\left(\frac{3 \pi^{2}}{2}\right)^{\frac{2}{3}} \rho^{\frac{2}{3}}+\frac{G_{\sigma}}{6 M^{2}}\left(\frac{3 \pi^{2}}{2}\right)^{\frac{2}{3}} \frac{\rho^{\frac{5}{3}}}{1+d G_{\sigma} \rho}+\frac{G_{\rho}}{8} \rho .
$$

If the Fock exchange is taken into account, the QMC Hartree-Fock symmetry energy for the nuclear matter becomes,

$$
\begin{aligned}
a_{\mathrm{sym}}^{\mathrm{HF}} & =\frac{\hbar^{2}}{6 M}\left(\frac{3 \pi^{2}}{2}\right)^{\frac{2}{3}} \rho^{\frac{2}{3}}+\frac{1}{3}\left(\frac{3 \pi^{2}}{2}\right)^{\frac{2}{3}}\left(\frac{G_{\omega}}{m_{\omega}^{2}}-\frac{G_{\sigma}}{m_{\sigma}^{2}}\right) \rho^{\frac{5}{3}} \\
& +\left[\frac{5 G_{\rho}}{32}+\frac{G_{\sigma}}{8\left(1+d \rho G_{\sigma}\right)^{3}}-\frac{G_{\omega}}{8}\right] \rho .
\end{aligned}
$$

In the SHF model, the symmetry energy for the nuclear matter has the following form [30],

$$
\begin{aligned}
a_{\mathrm{sym}}^{\mathrm{SHF}}= & \frac{\hbar^{2}}{6 M}\left(\frac{3 \pi^{2}}{2}\right)^{\frac{2}{3}} \rho^{\frac{2}{3}}-\frac{1}{24}\left(\frac{3 \pi^{2}}{2}\right)^{\frac{2}{3}}\left[3 t_{1} x_{1}-t_{2}\left(4+5 x_{2}\right)\right] \rho^{\frac{5}{3}} \\
& -\frac{1}{8} t_{0}\left(2 x_{0}+1\right) \rho-\frac{1}{48} t_{3}\left(2 x_{3}+1\right) \rho^{\alpha+1} .
\end{aligned}
$$

The symmetry energy contains two contributions: 1) from the kinetic energy; 2) from the interaction. It is seen that the QMC and SHF models have similar density-dependent forms for the kinetic energy part which originates from different Fermi momenta of nucleons and the momentum dependence of the isoscalar potential. The momentum dependence of the isoscalar potential has been well analyzed with experimental data [39, 40]. The different behavior of the density dependence in the symmetry energy would be caused by the interaction part which is from the isovector potential of the energy density functional. From Eq. (21) in the Hartree approximation, we see that the symmetry energy contributed from the interaction part, $\frac{G_{\rho}}{8} \rho$, has a linear form respecting the density which dominates the symmetry energy. The Fock exchange leads to a nonlinear density-dependent interaction part for the symmetry energy, see Eq. (22) for the QMC model. Fig. 7 displays the curves of the symmetry energy with and without the Fock exchange. It is seen that the inclusion of the Fock exchange gives a strong density-dependent symmetry energy. In the figure, we have also given the symmetry energy curve of the Hartree calculation with re-fitted parameters (readjust the QMC coupling constants by fitting the properties of the nuclear matter at the saturation) to see if the effect of the Fock exchange can be included by adjusting parameters. We see that the effect from 


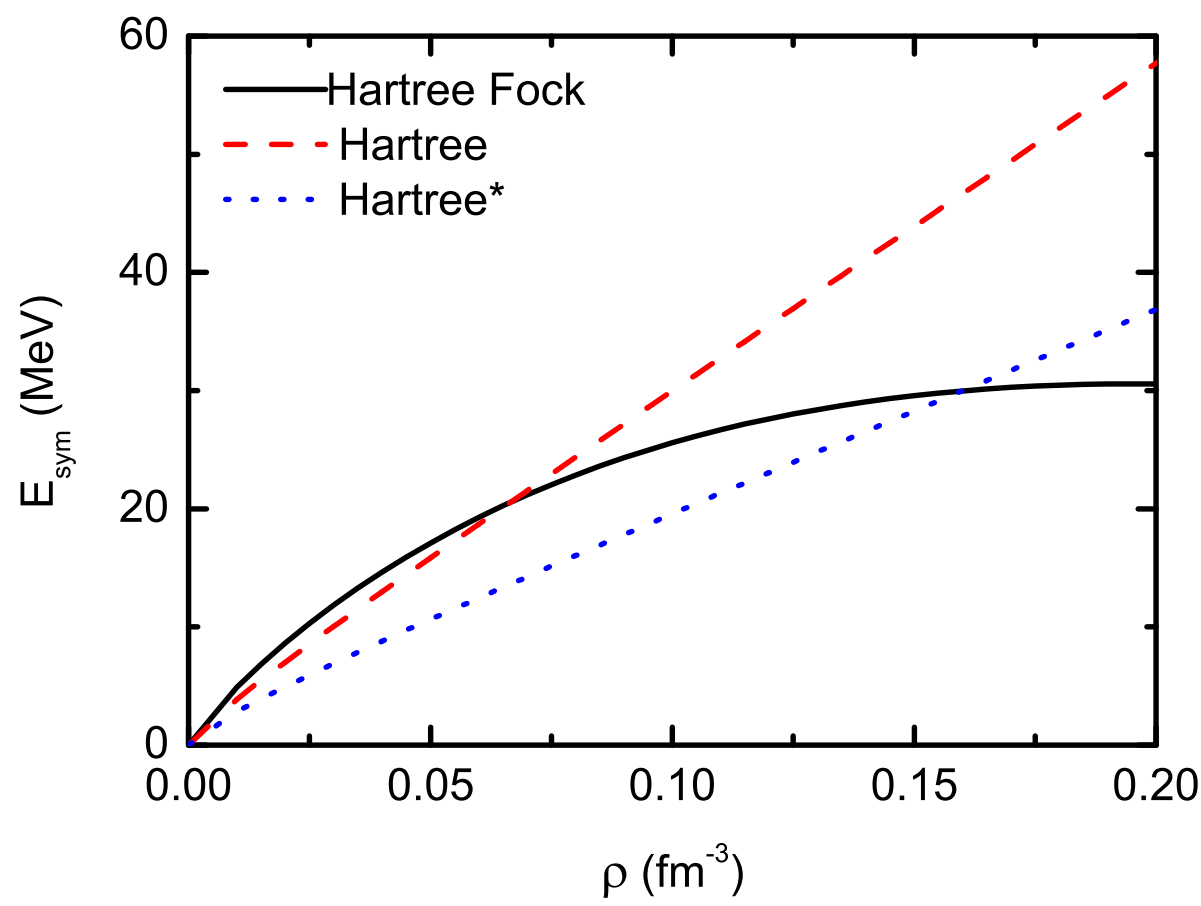

Figure 7. (Color online) Symmetry energy curves calculated by the QMC model in the Hartree and Hartree-Fock approximations. The dashed line is obtained by the Hartree approximation using the same parameter values as in the Hartree-Fock calculation. The dotted line is also for the Hartree calculation but with parameters readjusted by re-fitting nuclear matter properties at the saturation density. 


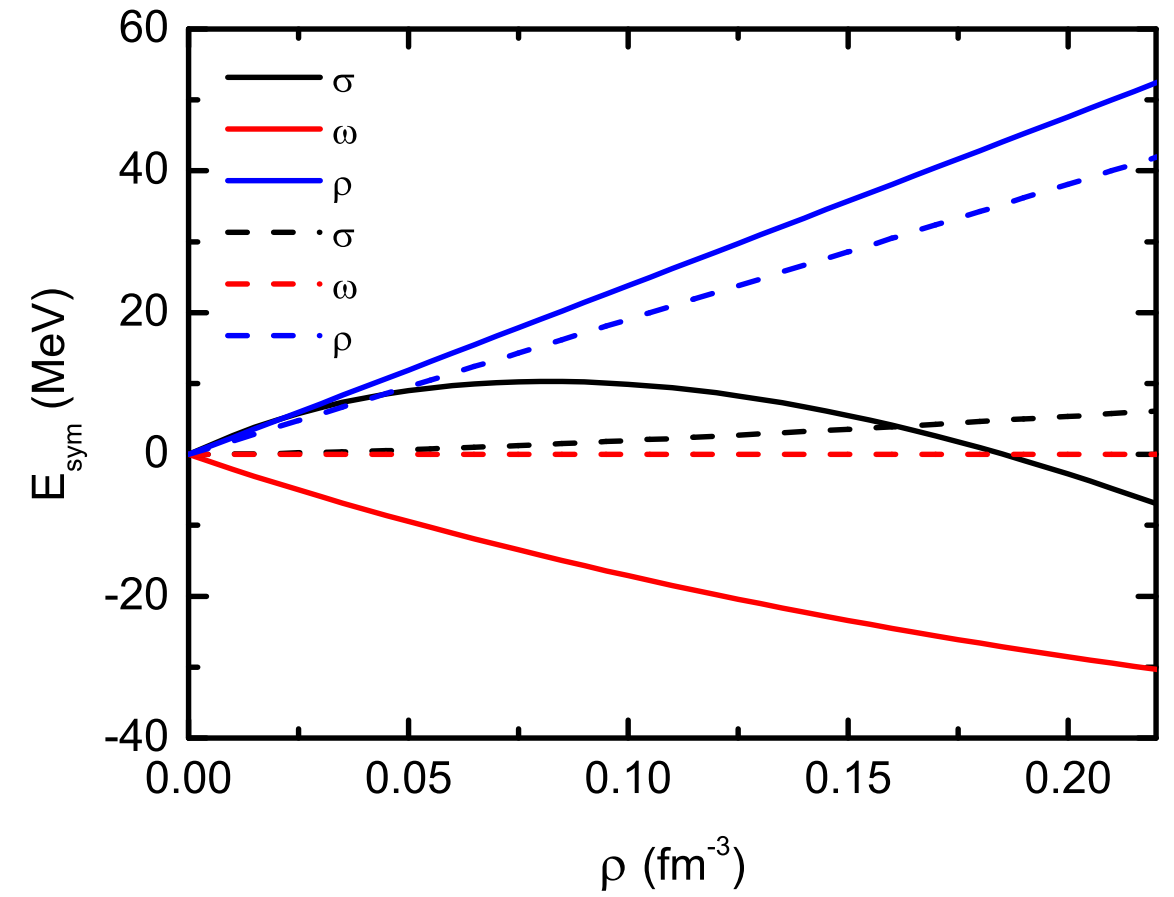

Figure 8. (Color online) Contributions of the $\sigma, \omega$, and $\rho$ mesons to the symmetry energy. The solid lines are for the QMC Hartree-Fock calculations, and the dashed lines are for the QMC Hartree calculations with the same parameters. 
the Fock exchange can not be included by readjusting parameters. It has been pointed out that models within the Hartree approximation give nearly-linear symmetry energy [16].

It is seen from Eq. (22) that the $\sigma$ meson brings a density dependent contribution to the interaction part of the symmetry energy through the Fock exchange. In Fig. 8, we show the contributions of the mesons to the symmetry energy in the QMC-Hartree and QMC-Hartree-Fock models. We see that, with the Fock exchange included, the contributions of the $\rho$ and $\omega$ mesons have nearly linear but opposite trends against the density. These two parts can be canceled partly. The $\sigma$ meson through the Fock exchange produces a strong density dependence, which increases first with density and then decreases drastically in higher density, leading to a soft behavior of the symmetry energy.

We further study effects on the symmetry energy from the density-dependent meson-nucleon exchange interaction. If the QMC model is compared with RMF model, one would find that the main difference is from the scalar field 41]. The scalar field in the QMC model has a nonlinear density dependence, which is derived by considering subnucleonic freedoms [24, 25, 41]. This nonlinear behavior originates from the scalar polarizability parameter $d$ of the nucleon. In the MIT bag model, it can be well represented by the bag radius [24, 28, 29]. Fig. 9 plots the density dependence of the symmetry energy with assuming the different values of the parameter $d$. The standard value of the parameter $d$ is $0.150 \mathrm{fm}$ which corresponds to a bag radius of $0.8 \mathrm{fm}[24$. If we set $d=0$ which is equivalent to assuming a point particle for the nucleons, the meson exchange interaction becomes density independent, which gives approximately linear symmetry energy, see Fig. 9. With increasing the parameter $d$, the symmetry energy becomes soft against the density. However, the Hartree calculation still give stiff linear symmetry energy. By comparing these calculations, we can conclude that the nonlinear scalar field can soften the symmetry energy curve through the Fock exchange interaction. As mentioned already in the introduction of the paper, the symmetry energy is still a debating problem. Different models give quite different predictions of the symmetry energy [5, 8, 10 18]. However, a soft symmetry energy seems to have been supported by the recent analysis of experimental data [11].

\section{Summary and conclusion}

With the inclusion of spin-exchange in the Skyrme force, we have discussed the relation between the Skyrme and meson-nucleon exchange forces. As in Ref. 24], the Skyrme force can be understood within the QMC model. By comparing the energy density functionals, a set of Skyrme parameters including the Skyrme spin-exchange parameters $x_{1}, x_{2}$ and $x_{3}$ is obtained, which can give reasonable results for the properties of nuclear matter and finite nuclei. However, the determination of the parameters is not the aim of the paper. Many sets of the parameters for the Skyrme force have existed with fitting to the experimental data of finite nuclei also. 


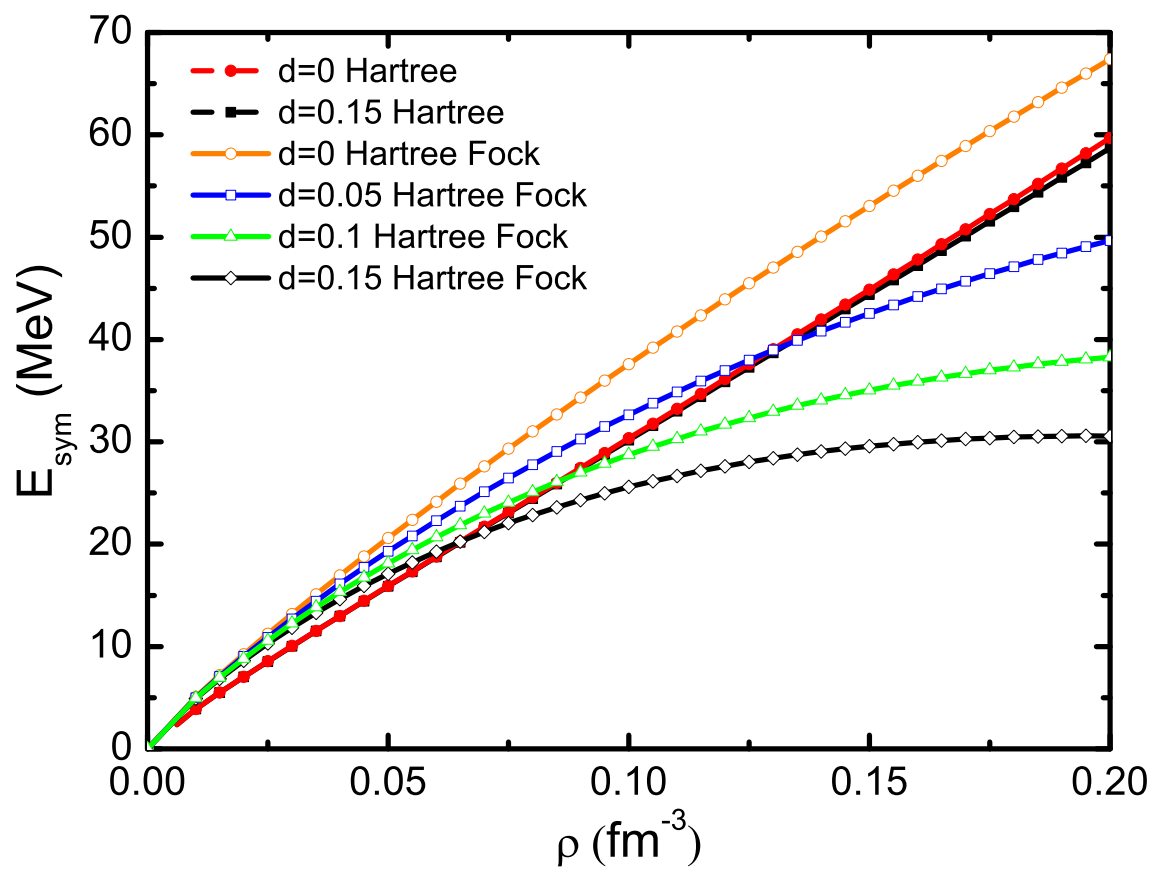

Figure 9. (Color online) Symmetry energy curves calculated by the QMC model in the Hartree and Hartree-Fock approximations with assuming different values of the parameter $d$. The dashed lines are obtained by the Hartree approximation and the solid lines are obtained by the Hartree-Fock calculation. All the calculations use the same parameters as QMC HartreeFock except the parameter $d$. 
We have paid special attention to the isovector channel of the nuclear energy density functionals. The QMC and SHF models have a similar density dependence for the isoscalar channel, but have different forms for the isovector channel. It is found that the Fock exchange leads to a nonlinear density-dependent isovector channel in the QMC model, which makes a soft symmetry energy. The nonlinear density dependence is produced by the scalar field which is derived by considering subnucleonic freedoms.

\section{Acknowledgement}

This work has been supported by the Chinese Major State Basic Research Development Program under Grant 2007CB815000 and the National Natural Science Foundation of China under Grant Nos. 10735010 and 10975006. C.Q. acknowledges the support from the Swedish Research Council (VR) under grant Nos. 623-2009-7340 and 2010-4723.

\section{References}

[1] M. Bender, P.-H. Heenen, and P.-G. Reinhard, Rev. Mod. Phys. 75 (2003) 121 , and references therein.

[2] W. Kohn, and L.J. Sham, Phys. Rev. 140 (1965) A1133.

[3] L. Brito, Ph. Chomaz, D.P. Menezes, and C. Providência, Phys. Rev. C 76 (2007) 044316.

[4] J.R. Stone and P.-G. Reinhard, Prog. Part. Nucl. Phys. 58 (2007) 587.

[5] B.A. Li, L.W. Chen, and C.M. Ko, Phys. Rep. 464 (2008) 113.

[6] D. Vautherin and D.M. Brink, Phys. Rev. C. 5 (1972) 626.

[7] B.A. Brown, Phys. Rev. C 58 (1998) 220.

[8] J.R. Stone, J.C. Miller, R. Koncewicz, P.D. Stevenson, and M.R. Strayer, Phys. Rev. C 68 (2003) 034324.

[9] P. Klüpfel, P.-G. Reinhard, T.J. Bürvenich, and J.A. Maruhn, Phys. Rev. C 79 (2009) 034310.

[10] A.E.L. Dieperink, Y. Dewulf, D.Van Neck, M. Waroquier, and V. Rodin, Phys. Rev. C 68 (2003) 064307.

[11] Z.G. Xiao, B.A. Li, L.W. Chen, G.C. Yong, and M. Zhang, Phys. Rev. Lett. 102 (2009) 062502.

[12] V.R. Pandharipande and V.K. Gaede, Phys. Lett. B 39 (1972) 608.

[13] B. Friedman and V.R. Pandharipande, Nucl. Phys. A 361 (1981) 502. 
[14] R.B. Wiringa, V. Fiks, and A. Fabrocini, Phys. Rev. C 38 (1988) 1010.

[15] P.G. Krastev and F. Sammarruca, Phys. Rev. C 74 (2006) 025808.

[16] L.W. Chen, C.M. Ko, and B.A. Li, Phys. Rev. C 76 (2007) 054316.

[17] B. Liu, V. Greco, V. Baran, M. Colonna, and M. Di Toro, Phys. Rev. C 65 (2002) 045201.

[18] Z.H. Li, U. Lombardo, H.J. Schulze, W. Zuo, L.W. Chen, and H.R. Ma, Phys. Rev. C 74 (2006) 047304.

[19] M.B. Tsang, Y.X. Zhang, P. Danielewicz, M. Famiano, Z.X. Li, W.G. Lynch, and A.W. Steiner, Phys. Rev. Lett. 102 (2009) 122701.

[20] S.R. Souza, M.B. Tsang, B.V. Carlson, R. Donangelo, W.G. Lynch, and A.W. Steiner, Phys. Rev. C 80 (2009) 041602(R).

[21] C. Fuchs and H.H. Wolter, Eur. Phys. J. A 30 (2006) 5.

[22] P.A.M. Guichon, Phys. Lett. B 200 (1988) 235.

[23] P.A.M. Guichon, K. Saito, E. Rodionov, and A.W. Thomas, Nucl. Phys. A 601 (1996) 349.

[24] P.A.M. Guichon, H.H. Matevosyan, N. Sandulescu, and A.W. Thomas, Nucl. Phys. A 772 (2006) 1.

[25] K. Saito, K. Tsushima, and A.W. Thomas, Prog. Part. Nucl. Phys. 58 (2007) 1.

[26] M.E. Bracco, G. Krein, and M. Nielsen, Phys. Lett. B 432 (1998) 258.

[27] G. Krein, A.W. Thomas, and K. Tsushima, Nucl. Phys. A 650 (1999) 313.

[28] J.R. Stone, P.A.M. Guichon, H.H. Matevosyan, and A.W. Thomas, Nucl. Phys. A 792 (2007) 341.

[29] P.A.M. Guichon and A.W. Thomas, Phys. Rev. Lett. 93 (2004) 132502.

[30] E. Chabanat, P. Bonche, P. Haensel, J. Meyer, and R. Schaeffer, Nucl. Phys. A 627 (1997) 710.

[31] J. Friedrich and P.-G. Reinhard, Phys. Rev. C 33 (1986) 335.

[32] P. Bonche, H. Flocard, and P.-H. Heenen, Comp. Phys. Comm. 171 (2005) 49.

[33] H. Euteneuer, J. Friedrich, and N. Voegler, Nucl. Phys. A 298 (1978) 452.

[34] J.P. Jeukenne, A. Lejeune, and C. Mahaux, Phys. Rep. 25 (1976) 83.

[35] V. Baran, M. Colonna, V. Greco, and M. Di Toro, Phys. Rep. 410 (2005) 335. 
[36] C. Xu, B.A. Li, and L.W. Chen, Phys. Rev. C 82 (2010) 054607.

[37] E. van Dalen, C. Fuchs, and A. Faessler, Phys. Rev. Lett. 95 (2005) 022302.

[38] C. Xu and B.A. Li, Phys. Rev. C 81 (2010) 064612.

[39] S. Hama, B.C. Clark, E.D. Cooper, H.S. Sherif, and R.L. Mercer, Phys. Rev. C 41 (1990) 2737.

[40] P. Danielewicz, Nucl. Phys. A 673 (2000) 375.

[41] S. Pal, M. Hanauske, I. Zakout, H. Stöcker, and W. Greiner, Phys. Rev. C 60 (1999) 015802. 